\title{
Measurement-based Pre-Assignment Scheme with Connection-level QoS Support for Multiservice Mobile Networks
}

\author{
Xiaoyuan $\mathrm{Luo}^{1}$, Ian $\mathrm{Thng}^{2}, \mathrm{Bo}^{3}{ }^{3}$ and Shengming Jiang ${ }^{3}$ \\ 1,2 Department of Electrical Engineering \\ ${ }^{4}$ Centre for Wireless Communication \\ National University of Singapore \\ E-mail: \{engp7708,eletlj,cwcjsm\}@nus.edu.sg \\ ${ }^{3}$ Department of Computer Science \\ Hong Kong University of Science and Technology \\ E-mail: bli@cs.ust.hk
}

\begin{abstract}
This paper contributes a new adaptive approach to prevent handoff failure due to lack of resources in adjacent cells in a mobile cellular network. Known as the Measurementbased Pre-assignment (MPr) technique, the technique is useful for implementation in micro/pico cellular networks which offers a Quality of Service (QoS) guarantee against call dropping. The technique is fully automated, simple, efficient, robust and generic for implementation. The notable feature is that the technique accepts different QoS specifications for different grades of handoff calls and ensures that these QoS levels are preserved under changing load conditions.
\end{abstract}

Subject Area: Handoff Support, Quality of Service, Cellular Networks

\section{Introduction}

The event of an unfinished call being terminated by the system is known to infuriate users much more compared to a new call that is unable to get through. In fact, one such experience is good enough to nullify any good opinions the user might have on a number of other previous calls that was successfully made. These rude terminations often occur at cell boundaries when a handoff request is being made to a cell that has insufficient resources. With cell sizes being systematically decreased to promote better frequency reuse which, by the same token, also increases handoff events, mechanisms that ensure that the probability of handoff dropping events does not exceed a specified QoS level is certainly useful to micro-cell wireless networks [1].

To reduce handoff failure due to lack of resources in adjacent cells, a basic approach is to reserve resources for handoffs in each cell. The well-known guard channel (GC) scheme and its variations $[2,3,4]$ reserve a fixed number of channels in each cell exclusively for handoffs in a single service environment. Generally, GC schemes are not adaptive to changing load conditions and no QoS guarantee is provided. A recent work [5] has shown that the guard channel scheme is unable to provide fair handoff support to different service types, since more bandwidth has to be reserved for wide bandwidth calls.

A variety of channel allocation strategies have been contributed for multi-service support in mobile networks. Based on multi-dimensional birth-death processes, the author in [5] developed 
a framework that is useful for teletraffic performance analysis and modelling for a broad class of problems stemming from cellular communication architecture. In [6, 7], two-tier resource allocation schemes for two types of incoming new calls were considered, a narrow-band type and a wide-band type. Complete sharing (CS), complete partitioning (CP), and hybrid allocation were investigated. But both schemes assume that only the narrow-band call can request handoffs. In [8], resource management for an integrated mobile networks with real-time connections and nonreal-time connections is broken up into two independent issues, namely, class-based wireless call admission control, and bandwidth allocation to admitted connections according to their quality of service requirement. However, non-real-time connections are assumed not to request for handoff and real-time connections are assumed to have the same bandwidth requirements. This limits the scope of the models.

The schemes surveyed above have focused more on channel allocation as opposed to handoff support which is more relevant in this paper. Existing techniques [9]-[17] utilises a combination of motion detection and load status broadcasting (to neighbouring cells) to support multi-service handoffs. Handoff support schemes using motion prediction models can be found in [9, 10, 11]. The shadow cluster concept [12], and its event-based variation [13], exploit the network capability to predict the motion probability of every mobile. The servicing base station must maintain status of each active call, and delivery it to all cells in the shadow cluster, i.e. the cells that the mobile might visit in the future. The efficiency of these schemes depends heavily on the accuracy of the network in predicting the potential movement of mobile hosts. The use of load status or number of active channels in neighbouring cells, to a determine local admission thresholds, are found in $[15,14,17]$, etc. These schemes create dependencies on neighboring cells for local call admission process, which may not do too well on the issue of robustness, for example, the possible event of a neighbouring cell who are no longer compliant to the information exchange process due to some unforeseen circumstances. Three problems remain unanswered in supporting handoff calls in a multi-service environment:

- Implementation complexity. Most schemes assume additional function to be performed by the network (e.g. signalling and motion prediction), and require base station to have high computation capability and large buffers to store call status or load status in neighbouring cells.

- The assignment of reserved channels is neglected. Most contributions have been focused on determining the total amount of resources to be reserved for all handoff calls. It is not clear which of the reserved channels are for which class of calls. If reserved resources are not distinguished for different call classes, wide-band calls will encounter much higher handoff dropping probabilities than narrow-band calls, as pointed out in [5] and illustrated later with numerical results.

- Complicated analytical model. Due to the modelling complexity of the multi-service system, a simple close-form formula to relate the desired QoS with the amount of resource to reserve is currently unavailable.

In this paper, we propose a Measurement Pre-reservation (MPr) scheme for the support of multiservice handoff with QoS guarantee on handoff dropping. The MPr scheme is adaptive to changing load conditions, changing amount of reserved resources dynamically. The reserved resources for each type of call with different QoS requirement is differentiated. A close-form solution relates the specified QoS levels with the amount of channels to reserve for each class of calls. This gives the wireless network provider full and easy control of the system even to the extent of changing the QoS level of any call type under widely varying load scenarios. Also, the MPr scheme preserves cell independence. It is a generic scheme that can be implemented in any cell even the adjacent cell is not compliant to the proposed scheme. It does not maintain the motion status for each call and there is no requirement for information exchanges amongst neighbouring cells. Consequently, issues leading to inefficiency of the system like late or delayed arrivals of inter-cell information 
cannot affect an MPr-compliant cell. Finally, the MPr scheme is practical for implementation as the operation for a base station is fairly simple.

This paper is organised as follows. We start from illustration of basic measurement-based pre-reservation for handoff a single service environment. Thereafter, we present the enhancement to support multiple QoS levels for different type of handoff calls in a multi-service environment. Finally, simulation results will be presented to illustrate the usefulness of the technique.

\section{The Pre-Assignment Methodology in MPr}

A fundamental difference between the GC scheme and the MPr scheme is the methodology of assigning the reserved channels. Figure 1 and 2 illustrates the resource management of the proposed MPr scheme, in comparison with the Guard Channel scheme, in a homogeneous call environment. The GC scheme employs a post-assignment methodology as illustrated in Figure 1 where handoff calls and new calls are first assigned to the common channels. In the event the common channels are depleted, new calls are blocked while handoff calls are assigned to the reserved channels. In the pre-assignment methodology of Figure 2 that is commensurate with the MPr technique, handoff calls are immediately assigned to reserved channels. If the reserved channels are depleted, handoff calls will have to compete with new calls for the common channels.

By using the pre-reservation metrology, the MPr scheme enjoys a desirable feature that a stated QoS can be easily "understood" and realised by the resource controller. The MPr scheme computes a reservation pool size for the stated QoS specification under the given loading conditions. It would also be clear later, that a smooth enhancement of the MPr scheme is found to support multiple QoS specification in a multi-service environment. In contrast, for a stated QoS, the number of Guard Channels to be reserved is cumbersome to determine, as it relies on state-transition queuing analysis as used in $[2,3]$ or looking up multiple tables with different loading parameters. Although the GC scheme is optimal for a evaluation function constructed by using the linear sum of call blocking probability and handoff dropping probability [14], the analytical complexity may not be desirable to guarantee a stated QoS under changing load conditions. For the same reason, extending the guard channel scheme for handoff support a multi-service environment will encounter considerable difficulty [5].

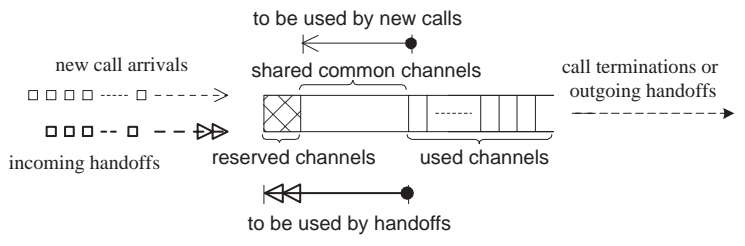

Figure 1: Post-assignment in a Guard Channel scheme

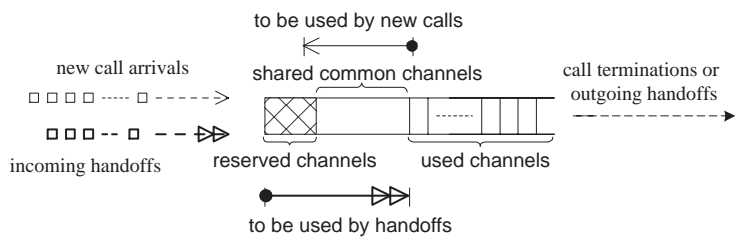

Figure 2: Pre-assignment in the proposed MPr scheme 
Two mechanisms are required for the MPr scheme. The first mechanism is described as follows: Given a target reservation pool size, i.e. $R_{R P S}$, which provides the required call dropping QoS specified for the service, a mechanism is required to maintain the number of reserved channels at time $t, R(t)$, towards $R_{R P S}$.

As an illustration, consider the arrival of a handoff call at time $t$. Due to the pre-assignment principle, the number of reserved channels will immediately shrink by one as follows:

$$
R\left(t^{+}\right)=R\left(t^{-}\right)-1<R_{R P S}
$$

The second mechanism is, to determine $R_{R P S}$ in order to satisfy the desired call dropping QoS for the cell. The design of the second mechanism is rather challenging as it is constrained by the following factors:

- The mechanism is adaptive towards changing load conditions, shrinking/enlarging where necessary to ensure the specified call dropping QoS for the cell.

- The mechanism can be deployed in the network in a distributed fashion where the mechanisms run independently of each other from cell to cell.

- For generic and robust implementation, the mechanism should not require information exchanges amongst neighbouring cells.

- The mechanism should also support multiple QoS requirements for different types of services.

In the following section, we present the two mechanisms required to perform MPr in a wireless cellular network that is operating in a homogeneous call environment.

\section{MPr for a Homogeneous Call Environment}

A typical homogeneous service environment often assumes that each call, i.e. handoff call or new call, occupies one unit of bandwidth, or a channel. It is also assumed that new call arrivals and handoff call arrivals are Poisson processes $[3,4]$. In addition, the call life time and call dwelling time within a cell are assumed to be exponentially distributed.

\section{$3.1 \quad$ Token-property Update of $R(t)$}

As mentioned before, a mechanism is required to update $R(t)$ (the number of reserved channels at time $t$ ) towards a given $R_{R P S}$ (the reservation pool size). Figure 3 illustrates the update logic being adopted for the MPr algorithm.

Figure 3 illustrates a very important property of the MPr scheme which we refer to as the token-property update. It is described as follows:

A channel can only be reserved at time $t$ if the following three conditions are true.

- At time $t^{-}$, the channel was in the busy state, servicing a call.

- At time $t^{-}, R\left(t^{-}\right)<R_{R P S}$.

- At time $t$, the above channel is released by the call due to termination or handoff.

The token-property update, which will be more meaningful later, also indicates the following:

- A channel that has been designated to the common pool cannot transit directly to the reservation pool. It has to transit a busy state first before it can be considered for designation in the reservation pool. See Figure 3. 


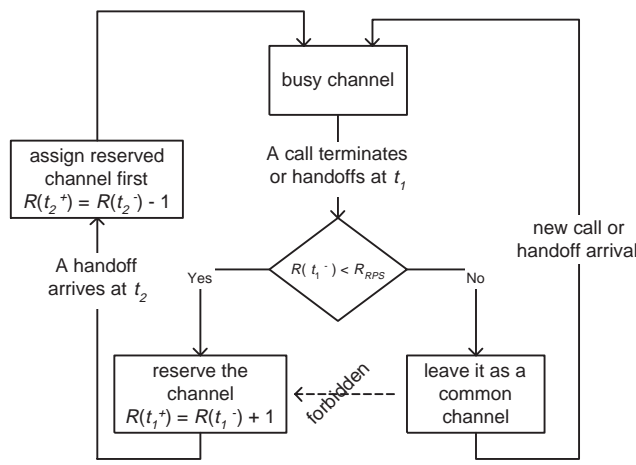

Figure 3: State transition of a radio channel

- If the number of reserved channels have not reached the desired the number, i.e. $R\left(t^{-}\right)<R_{R P S}$ , then a channel returned to the system, when a call terminates or handoffs, is immediately reserved.

- If the reservation pool is depleted, i.e. $R(t)=0$, then a handoff call at time $t$ will have to compete with new calls for an idle channel in the common pool. A strict dropping policy may be employed, which drops a handoff request under such condition, but is not encouraged for the same reasons as discussed in Section 1. Comparison of such a variation with the normal admission condition for handoff calls will be presented in the numerical section.

The token-property update policy holds the key to the simplicity of the MPr scheme and contributes, to a certain extent, some of the favourable features of the MPr scheme. This will be elaborated further in the next section.

\subsection{Update of $R_{R P S}$}

With the token-bucket update policy of $R(t)$ in place, it is now rather easy to develop a token bucket model for updating $R_{R P S}$ as illustrated in Figure 4. In the figure,

- The size of the token bucket represents the $R_{R P S}$, i.e. the reservation pool size.

- Arriving tokens at the bucket ingress represent events where channels are released (by call handoff or call termination).

- If the bucket is full, i.e. $R(t)=R_{R P S}$, then any arriving tokens are discarded, meaning that a released channel is designated to be a common channel.

- Arriving handoff calls are assigned reserved channels if tokens are available. If the token bucket is empty of tokens (i.e. reservation pool is depleted), then there is no guarantee the handoff call will be admitted as it depends on the availability of common pool channels.

The token bucket is essentially a M/M/1 queue system with finite capacity $R_{R P S}$. We shall see that the possibility for a handoff to be accepted when there is no channel reserved is fairly low in the proposed MPr scheme, although it is possible. Such case requires some conditions to be satisfied. Firstly, the free channel must be released at the time the reservation pool was full so that it was not captured. Another necessary condition is that during the period handoff calls move in and use up all the reserved channels, no new call attempts within the radio cell utilise that particular free 


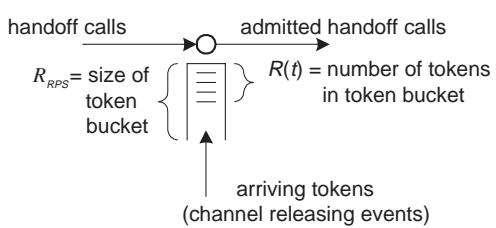

Figure 4: The analytical model of the MPr scheme

channel. Therefore, the probability that the reservation pool is empty effectively gives a tight upper bound for call dropping probability.

If the call dropping QoS probability is specified to be $P_{Q a S}$, then the MPr scheme has to determine a suitable token bucket size to ensure that

$$
P_{Q a S}=P(\text { handoff call is droppped }) \leq P \text { (token bucket is empty) }
$$

Employing the $\mathrm{M} / \mathrm{M} / 1 / \mathrm{k}$ queue principles [18], we note that

$$
\begin{aligned}
& P(\text { token bucket is empty })=\frac{1-\rho}{1-\rho^{R_{R P S}-1}} \\
& \text { where } \quad \rho=\frac{\text { channel releasing rate }}{\text { incoming handoff rate }}=\frac{\mu_{r}}{\lambda_{h}}
\end{aligned}
$$

Incorporating the above equations, we obtain the update condition for minimum $R_{R P S}$ as follows,

$$
R_{R P S} \geq \frac{\ln \frac{P_{Q o S}+\rho-1}{P_{Q o S}}}{\ln \rho}-1
$$

It is noted that the update for $R_{R P S}$ in 5 only requires local cell metrics $\mu_{r}$ and $\lambda_{h}-$ this is precisely the strength of the MPr scheme in terms of robustness and generic deployment in a wireless network where not all cells are MPr compliant.

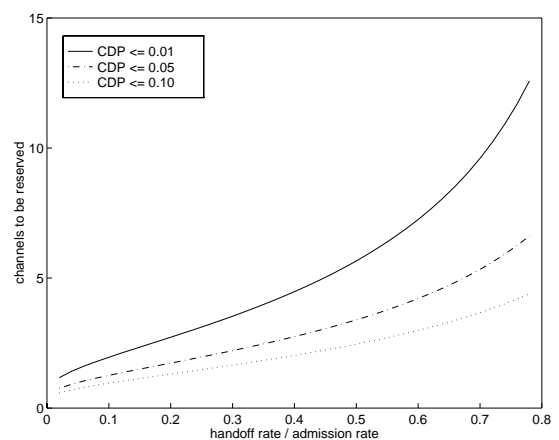

Figure 5: Channels to be reserved over $1 / \rho$

\subsection{Fine Tuning of the $R_{R P S}$ Update}

There are a number of issues regarding the update equation of $R_{R P S}$ in 5 , the first being the estimate of the channel releasing rate $\mu_{r}$ which can be replaced with

$$
\mu_{r}=\lambda_{a}=\text { successful call admittance rate }
$$


Therefore there is no necessity to incorporate a MPrarate device to monitor the channel releasing rate as the call admission control device of the cell can indirectly perform that function.

It is now clear that the $1 / \rho$ represents the percentage of incoming handoff calls in the total successful call attempts, provided handoff failure is negligible because of the reserved resources. By monitoring $\rho$ in $5, R_{R P S}$ can be adaptively adjusted. This is seen more clearly in Figure 5 which plots $R_{R P S}$ against $1 / \rho$ for various $P_{Q o S}$. Notice that as the percentage of handoff rate increases, so does the number of channels to be reserved. The more stringent the QoS requirement, the more channels will be reserved.

In addition, there is no stringent requirement on the refresh frequency of $\lambda_{a}$ and $\lambda_{h}$, neither the identical update period for every cell in [15], nor the periodical flooding of call status to neighboring cells as in $[13,13]$. Once every few minutes should be adequate. Chances are that channels are reserved but handoff attempts decrease afterwards, e.g., when attendants are seated and a conference starts, the arrival rate would decrease drastically. In such a case, excessively reserved channels will be released upon the above mentioned measurement is refreshed and new size of the reservation pool is re-computed. To smooth out random or drastic measurement inaccuracies, an exponential smoothing average formulae can be employed to obtain a moving average $\lambda_{\text {ave }}$ as follows:

$$
\lambda_{\text {ave }}(n)=\alpha \lambda_{\text {ave }}(n-1)+(1-\alpha) \lambda_{\text {new }}
$$

each time a new rate $\lambda_{\text {new }}$ is measured.

\section{MPr Support for Multi-Service Handoff Calls}

In a multi-service environment with mixed call types, either of the following reasons require the cell to differentiate handoffs belonging to different call types: $i$ ) Different calls $i$ have different QoS requirement in terms of handoff dropping, i.e. $P_{Q a S i}$. $\left.i i\right)$ Different calls $i$ have different channel requirements $B_{i}$.

The MPr algorithm is amenable for application in both a complete partitioning environment and a resource sharing environment. Other bandwidth partitioning strategies that allocate bandwidth fairly for different traffic classes while maximising network throughput can be found in [8, 19].

\subsection{Multi-Service with Complete Partitioning Resource Allocation}

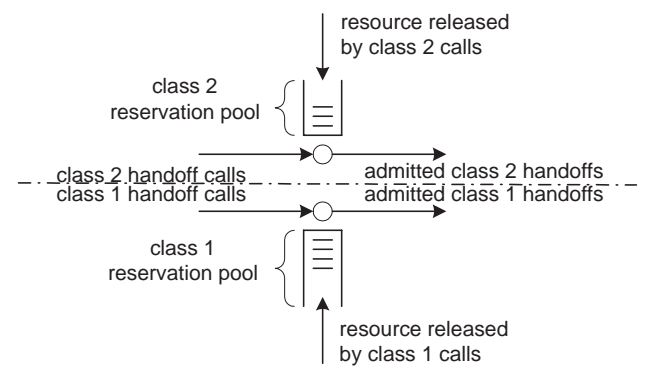

Figure 6: A complete partitioning multi-service environment

With complete partitioning (CP) resource allocation, the allocated bandwidth boundary for each service class is clearly demarcated. Thus an incoming class $i$ call consumes bandwidth allocated for class $i$ and an outgoing class $i$ call returns the resources back to its class. Consequently, the handoff reservation pool for each class is also separated from each other and belongs strictly to each class. In this environment, independent MPr mechanisms outlined in Section 3 can be used to 
update the required reservation pool for each class as shown in Figure 6. Thus the MPr mechanism in this scenario is just a trivial extension of the one in the homogenous call environment. A point to note is that 5 should take into consideration the different amount of bandwidth each class of call requires. Thus Equation 5 should be modified to

$$
R_{R P S_{i}} \geq B_{i} \times\left(\frac{\ln \frac{P_{Q o S_{i}}+\rho_{i}-1}{P_{Q o S_{i}}}}{\ln \rho_{i}}-1\right)
$$

where $R_{R P S_{i}}$ is the number of channels to be reserved in class $i$ and

$$
\begin{aligned}
\rho_{i} & =\frac{\mu_{r i}}{\lambda_{h i}}=\frac{\text { call releasing rate for class } i}{\text { incoming handoff rate for class } i} \\
& =\frac{\lambda_{a i}}{\lambda_{h i}}=\frac{\text { successful call admittance rate for class } i}{\text { incoming handoff rate for class } i}
\end{aligned}
$$

\subsection{Multi-Service Resource Sharing Environment}

In a resource sharing multi-service environment, the bandwidth of the cell is not demarcated and is shared by different call types. In order to support the different handoff requirements, a total of $i$ independent MPr processes is required, each maintaining their own respective reservation pools for each class $i$ as illustrated in Figure 7. An incoming class $i$ handoff call will immediately be assigned reserved channels corresponding to its class. And as before, whichever released channels not reserved (i.e. the rightful token buffer is full) merely becomes part of the pool of common channels.

However, a difficulty is now to decide which token buffer in Figure 7 deserves the arriving tokens (arising out of resource releasing events). Specifically, the difficulty lies with characterising the overall channel releasing process and designing an appropriate scheduling mechanism that preserves the $\mathrm{M} / \mathrm{M} / 1 / \mathrm{k}$ model for each reservation pool class. The difficulty can be overcome by the use of a poorer performing fictitious model rather than the actual model. Then based on the fictitious model, an appropriate and simple dispatching mechanism is described which is also applicable for use in the original model (which is better performing). Finally the appropriate update equations for the various reservation pool classes of the original model are provided.

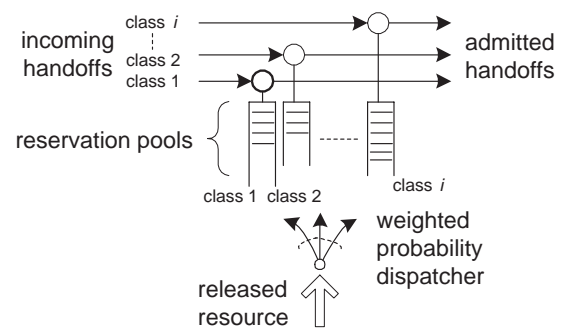

Figure 7: A resource sharing multi-service environment

\subsubsection{The Overall Channel Releasing Process}

The overall channel releasing process associated with Figure 7 is not a conventional Poisson process but a sum of Poisson processes with bulk arrivals. The class $i$ call completion or outgoing-handoff process would return resource to the system at the rate of $\mu_{r i}$ with bulk arrival $B_{i}$, where $B_{i}$ is units of bandwidth used by class $i$ calls. Characterising the overall channel releasing process is, 


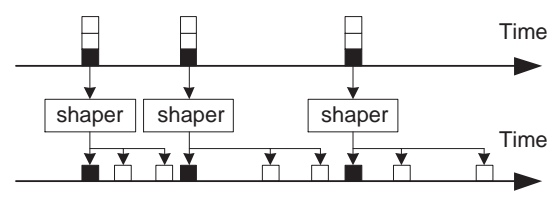

Figure 8: Shaping the bulk arrival into a more inferior system

in general, rather difficult, but the difficulty can be overcome by considering a inferior fictitious system.

Due to the memory-less nature of the Poisson process, a conventional Poisson process at the rate $\mu_{r i}^{\prime}$ can be constructed from a given bulk arrival Poisson process with rate $\mu_{r i}$ and bulk size $B_{i}$ via shapers, which distribute/delay exponentially (in time) the bulk arriving channels, as illustrated in Figure 8. If we subject all the bulk arrival channel releasing processes of the cell to be exponentially shaped to conventional Poisson processes, then the overall channel releasing process of the fictitious model is a sum of conventional Poisson processes. Consequently, the overall channel releasing process of the fictitious model is a well characterised conventional Poisson process with rate $\mu_{r}$ given as follows:

$$
\mu_{r}^{\prime}=\sum_{i} \mu_{r i} B_{i}=\sum_{i} \lambda_{a i} B_{i}
$$

where $\lambda_{a i}$ is defined in 6 .

The model is fictitious since, in order to distribute the bulk arrivals to exactly construct a conventional Poisson process, shapers ought to have prior knowledge of future bulk arrival events - which is impractical. Nonetheless, the fictitious domain is useful for further analysis, not only because its overall channel releasing process is well characterised, but it is noted that in the fictitious system, its channel reservation process is inferior compared to the actual system's reservation process since shapers in the fictitious system introduce artificial delays to arriving resources that can otherwise be immediately reserved. Thus by assuming the fictitious domain characterised by 10 , it is clear that the eventual MPr update equations should perform at least equal or better in the actual system since the bulk arriving channel releasing processes are not delayed.

\subsubsection{Update of $R_{R H S i}$}

With the overall channel releasing process characterised, we designed a simple scheduling algorithm to dispatch the released resources to one of reservation pools for call classes which require handoff support. Referred to as the Weighted Probability Dispatcher, it is described as follows:

Weighted Probability Dispatcher: For each unit of channel returning to the system, the dispatching mechanism chooses token buffer $i$ with probability $B_{i} \lambda_{h i} / \sum_{i} B_{i} \lambda_{h i}$ as the rightful token buffer.

If the rightful class $i$ reservation pool is not full, the released channel is immediately reserved as a class $i$ channel. Otherwise the channel is relegated as a common pool channel. By using this dispatching mechanism, it is clear that the channel refilling rate of the reservation pool for class $i$ handoffs is as follows:

$$
\mu_{r i}^{\prime}=\mu_{r}^{\prime} \times \frac{B_{i} \lambda_{h i}}{\sum_{i} B_{i} \lambda_{h i}}
$$

where $\mu_{r}^{\prime}$, as defined in 10 , is the overall rate of the channel releasing process associated with the fictitious system.

For class $i$ handoff calls with bandwidth requirement $B_{i}$, the token refilling rate is $\mu_{r i}^{\prime} / B_{i}$, by counting $B_{i}$ unit of bandwidth as one token. 
Therefore, the appropriate reservation pool size to support class $i$ handoff calls with QoS requirement in terms of worst handoff failure probability $P_{Q a S}$ is

$$
R_{R P S i}=B_{i} \times\left(\frac{\ln \frac{P_{Q O S_{i}}+\rho_{i}^{\prime}-1}{P_{Q o S_{i}}}}{\ln \rho_{i}^{\prime}}-1\right)
$$

where

$$
\rho_{i}^{\prime}=\frac{\lambda_{h i}}{\mu_{r i}^{\prime} / B_{i}}=\frac{\lambda_{h i} B_{i}}{\mu_{r i}^{\prime}}
$$

In summary, by the inclusion of the Weighted Probability Dispatcher and the periodic updating of $\lambda_{h i}$ and $\lambda_{a i}$, i.e. loading conditions, the required reservation pool sizes for the various class of services can be adaptively adjusted by virtue of 12 . This sums up the MPr scheme for handoff support in a resource sharing Multi-Service environment. Finally, it is noted that in the MPr scheme of Figure 7, the reservation pools are clearly distinguished for the different class of services.

\section{$5 \quad$ Numerical Results}

The simulation model used here is a hexagonal system with three rings of radio cells as shown in Figure 9. We tested two scenarios, one for single service and the other with two call types. Both the call life time and channel holding time are set to be exponentially distributed. We tag individual cells and obtain performance metrics by simulation. Note the generic feature of the MPr scheme that there is no requirement that all cells must be MPr-compliant.

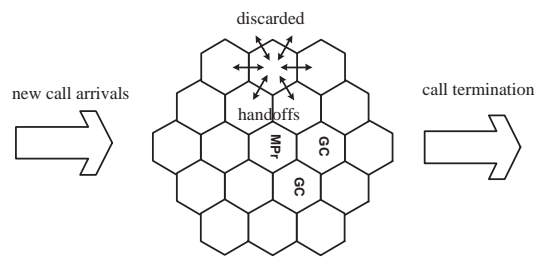

Figure 9: A cellular system with three rings of radio cells

\subsection{A Homogeneous Call Scenario}

Figure 10 shows the call dropping probability $(C D P)$ and call blocking probability $(C B P)$ of the MPr scheme with a target $C D P$ below 0.05 for a homogenous call environment. Each call occupies 1 unit of bandwidth. The total capacity for a base station is 30 . The mean call lifetime is 200 s and the mean channel holding time for a call to stay in one cell is 100 s.

For comparison, we present the results of the GC scheme with 2 and 3 guard channels, as they achieve comparable performance in terms of call dropping probability as well as call blocking probability, under light or mild load condition. When load increases, the CDP of GC scheme soars. However, the MPr scheme persists in keeping the CDP below the target level. By ensuring the handoff dropping QoS, a tradeoff occurs where the corresponding blocking probability of the MPr scenario is higher than either guard channel scenarios, because more channels are reserved for handoff calls. It has been proved

It should also be noted that for a stated QoS specification on call dropping, the MPr scheme is self-driven while the GC scheme requires either table lookup or two dimensional state-transition analysis as presented in $[2,3]$. 


\subsection{The Strict Dropping Policy}

The performance of the strict dropping policy in a homogeneous call evironment is also presented in Figure 11. In strict dropping policy, handoff calls are accepted only when there are channels available in the reservation pool, whereas in normal dropping policy, handoffs are accepted as long as there are unused channels. The strict dropping shows a worse case than the normal admission condition for handoff calls. It proves that the correct amount of resources is reserved to guarantee the QoS specification against handoff dropping by the MPr scheme. The lower call dropping probability for the normal admission under low load condition results from the competition for unused common channels, which are not guaranteed to be availabe under all loading conditions. Although it is shown in Figure 11a the expected QoS on call dropping is not violated, the strict dropping policy is not encouraged for the same reasons as discussed in Section 1.

\subsection{A Multi-service Scenario}

We tested a multi-service scenario with two call types. Both types of call may use all the available channels for the cell. The case of the complete partitioning environment is not presented as it is a trivial extension of the single-service scenario. The specifications of the two call types are shown in Table 1. Note that the wide-band call arrival rate is four times smaller than a narrow-band call and its handoff QoS requirements more stringent than the narrow-band call. Also note that the two types of calls have different motion characteristics.

\begin{tabular}{|c||c|c|}
\hline Call Parameters & Type I & Type II \\
\hline mean call life time & $300 \mathrm{~s}$ & $300 \mathrm{~s}$ \\
\hline mean dwelling time & $100 \mathrm{~s}$ & $150 \mathrm{~s}$ \\
\hline bandwidth reqrirement & 1 & 12 \\
\hline call arrival rate & $\lambda_{1}$ & $\lambda_{2}=0.25 \lambda_{1}$ \\
\hline target maximum call dropping & 0.10 & 0.05 \\
\hline
\end{tabular}

Table 1: Parameters for two types of calls

Figure 12 illustrates the call dropping probabilities obtained using the MPr scheme in comparison with GC schemes. We notice the overwhelming favouring of narrow-band calls compared to wideband calls in the GC cells. However, the MPr compliant cells do not over-favour the narrow-band calls, but maintain the stated QoS against handoff dropping for each type of calls, i.e. 0.10 for narrow-band calls in Figure 12(a) and 0.05 for wide-band calls for all loading conditions Figure $12(\mathrm{~b})$.

\section{Conclusion}

In this paper we presented a novel measurement-based pre-reservation scheme, known as MPr, to reduce handoff failure in mobile multimedia networks with mixed-call types. It is capable of supporting multiple connection-level QoS for handoff calls of different types. In addition, the MPr scheme is very simple, efficient, and amenable for implementation in any heterogeneous cellular network where not all cells are MPr compliant.

\section{References}

[1] A.S. Acampora, and M. Naghshineh, "Control and Quality-of-Service provisioning in high speed microcellular networks", IEEE Personal Communications, vol. 1, no.2, 1994.

[2] E. C. Posner and R. Guerin, "Traffic Policies in Cellular Radio that Minimize Blocking of Handoff Calls", ITC-11, Kyoto, 1985 


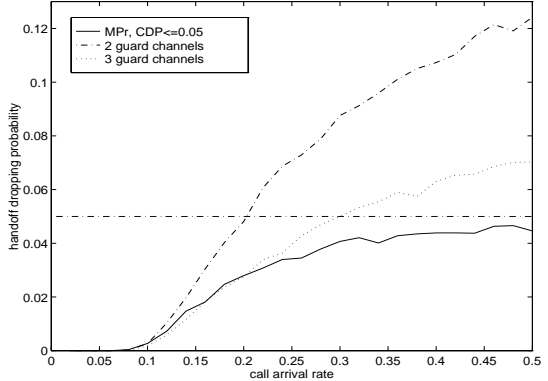

(a)

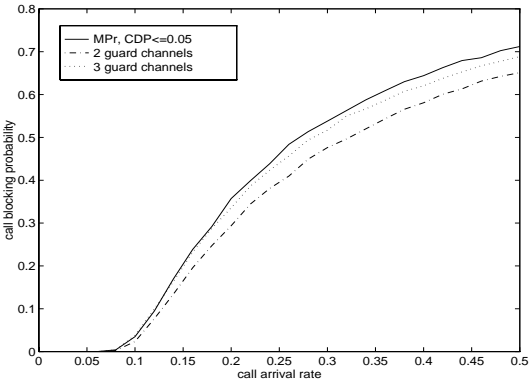

(b)

Figure 10: $C D P$ and $C B P$ v.s. arrival rate, single service

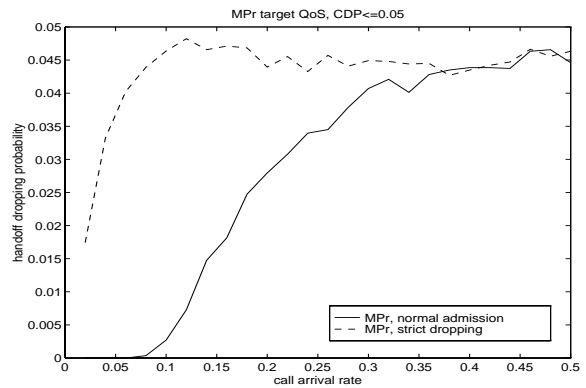

(a)

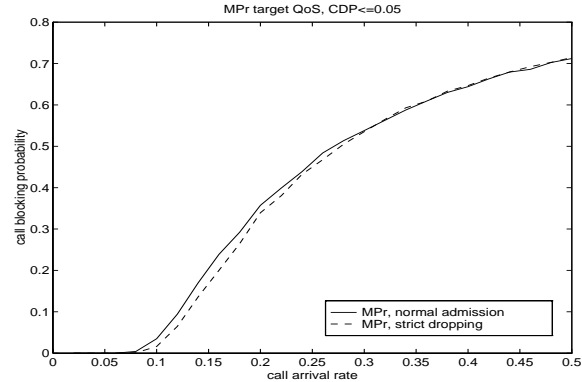

(b)

Figure 11: The strict dropping policy and normal admission for handoff

[3] D. Hong and S. S. Rappaport, "Traffic Model and Performance Analysis for cellular mobile radio telephone systems with prioritized and non-prioritized handoff procedures", IEEE Trasactions on Vehicular Technology, vol. 35, 1986, no. 3, pp. 77-92.

[4] Sirin Tekinay, Bijan Jabbari "A Measurement-Based Prioritization Scheme for Handovers in Mobile Cellular Networks", IEEE JSAC, vol 10, No. 8, October 1992.

[5] Stephen S. Rappaport and C. Purzynski, "Prioritised resource assignement for mobile cellular communication systems with mixed services and platform types", IEEE Trans. Veh. Tech., Vol.45, No.3, 1996.

[6] B. Epstein and M. Schwartz, "Reservation Strategies for Multi-Media Traffic in a Wireless Environment", IEEE Vehicular Technology Conference 1995, pp.165-169, 1995.

[7] J. Chen, M. Schwartz, "Reservation strategies for multi-media traffic in a wireless environment, performance summary", PIMRC'95, 1995

[8] M. Naghshineh, A. S. Acampora, "QoS Provisioning in Micro-Cellular Supporting Multimedia Traffic", INFOCOM 1995 


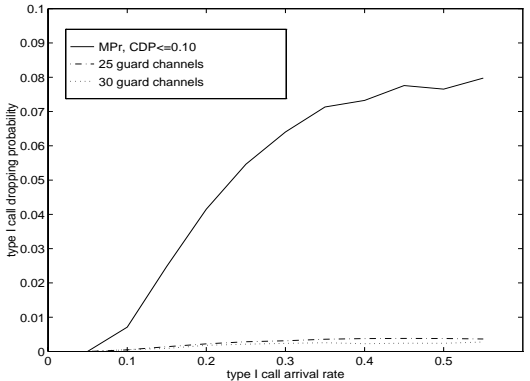

(a)

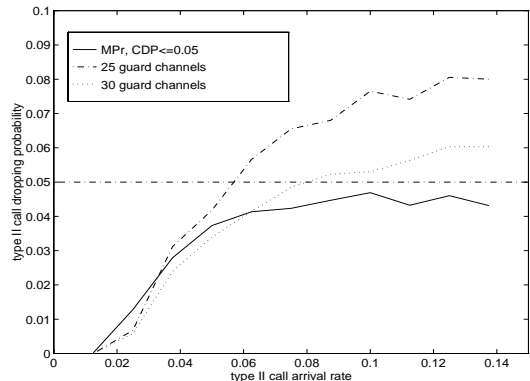

(b)

Figure 12: $C D P$ v.s. arrival rate, two types of calls

[9] A. Aljadhai, T. F. Znati, "A Predictive Bandwidth Allocation Scheme For Multimedia Wireless Networks".

[10] Zhuang, W.; Chua, K.C.; Jiang, S.M., "Measurement-Based Dynamic Bandwidth Reservation Scheme for Handoff in Mobile Multimedia Networks", IEEE ICUPC '98. Vol 1, pp311-315

[11] M. Zonoozi,and P. Dassanayake, "User Mobility Modeling and Characterization of Mobility Patterns", IEEE JSAC, Vol. 15, No. 7, September 1997, pp. 1239-1252

[12] D. A. Levine, I.F. Akyildiz and M. Nagshineh, "A Resource Estimation and Call Admission Algorithm for Wireless Multimedia Networks Using The Shadow Cluster Concept", IEEE/ACM Transaction on Networking, Vol. 5, No.1, February 1997, pp1-12.

[13] J. Misic, S. T. Chanson, F. S. Lai, "Admission Control for Wireless Networks with Heterogeneous Traffic using Event Based Resource Estimation", ICCCN 1997. IEEE, Piscataway, NJ, USA,97TB100187.. p 262-269

[14] R. Ramjee, R. Nagarajan, and Don Towsley, On Optimal Call Admission Control in Cellular Networks, INFOCOM'96, pp43-50.

[15] M. Nagshineh and M. Schwartz, "Distributed Call Admission Control in Mobile/Wireless Networks”, IEEE JSAC, Vol 14, No. 4, May 1996, pp711-716

[16] Chi-chao Chao, Wai Chen, "Connection admission control for mobile multiple-class personal communications networks", IEEE JSAC v15 n8 Oct 1997. p1618-1626.

[17] C. Oliveira, J.B. Kim, T. Suda, "An Adaptive Bandwidth Reservation Scheme for High Speed Multimedia Wireless Networks", IEEE JSAC v16 n6 Aug 1998. p 858-874

[18] Alberto Leon-Garcia, Probability and Random Processes for Electrical Engineering, 2nd Edition, Addison-Wesley.

[19] Y.H. Kim, and C. K. Un, "Analysis of Bandwidth Allocation Strategies with Access Restriction in Broadband ISDN", IEEE Transactions on Communication, Vol. 41, No.5 May 1993, pp. 771781 